\title{
WEAK SOLUTIONS TO THE INITIAL BOUNDARY VALUE PROBLEM FOR A SEMILINEAR WAVE EQUATION WITH DAMPING AND SOURCE TERMS
}

Abstract. We show local existence of solutions to the initial boundary value problem corresponding to a semilinear wave equation with interior damping and source terms. The difficulty in dealing with these two competitive forces comes from the fact that the source term is not a locally Lipschitz function from $H^{1}(\Omega)$ into $L^{2}(\Omega)$ as typically assumed in the literature. The strategy behind the proof is based on the physics of the problem, so it does not use the damping present in the equation. The arguments are natural and adaptable to other settings/other PDEs.

1. Introduction. Consider the boundary value problem

$$
(\mathrm{SW})\left\{\begin{array}{l}
u_{t t}-\Delta u+f(x, t, u)+g\left(x, t, u_{t}\right)=0 \quad \text { a.e. }(x, t) \in \Omega \times[0, \infty) ; \\
u(x, t)=0 \text { a.e. }(x, t) \in \partial \Omega \times[0, \infty) ; \\
\left.\left(u, u_{t}\right)\right|_{t=0}=\left(u_{0}, u_{1}\right) \quad \text { a.e. } x \in \Omega,
\end{array}\right.
$$

where $\Omega$ is a connected bounded domain with $C^{2}$ boundary. In [13] we proved local existence of solutions to the Cauchy problem $\left(\Omega=\mathbb{R}^{n}\right)$ under a set of assumptions $\mathrm{A}_{f}, \mathrm{~A}_{g}$ concerning the nonlinearities $f$ and $g$ that we will list below. In this work we are interested in solving the problem on a bounded domain, issue which is of great interest in control theory $([9,10])$. This paper complements the work in [13] as we will prove that the techniques of [13] apply to bounded domains as well; however, some nontrivial arguments have to be used in order to deal with the boundary conditions.

1.1. Assumptions. Here we present the list of assumptions that govern our result. Note that (A0)-(A8) concern the source and the damping terms, while (A9) is a geometric assumption on the boundary.

2000 Mathematics Subject Classification: Primary 35L05; Secondary 35L20.

Key words and phrases: wave equation, local existence, finite speed of propagation, nonlinear damping, interior source. 
(A0) $f$ is measurable in $x$, differentiable in $t$ a.e., differentiable in $u$ a.e., and there exists a continuous function $k$ such that for a.e. $x, t$,

$$
\left|f_{u}(x, t, u)\right| \leq k(r) \quad \text { for a.e. }|u| \leq r .
$$

(A1) Growth conditions on the source term $f$ :

(i) $f(x, t, 0)=0$;

(ii) $|f(x, t, u)| \leq m_{1}|u|^{p}+m_{2}|u|^{q}$ for some $1<q<p<2^{*}-1$ and $m_{1}, m_{2}>0$, where $2^{*}=2 n /(n-2)$.

(A2) $\left|f_{t}(x, t, u)\right| \leq K$ for some $K>0$.

$(\mathrm{A} 2)^{*} f$ does not depend on $t$ and $F(x, u)=\int_{0}^{u} f(x, v) d v \geq 0$.

(A3) $g=g(x, t, v)$ is measurable in $t$, differentiable in $x$, and continuous in $v$.

(A4) For every $x, t$ the function $v \mapsto g(x, t, v)$ is increasing and $g(x, t, 0)$ $=0$.

(A5) $v g(x, t, v) \geq C_{1}|v|^{m+1}$ and $|g(x, t, v)| \leq C_{2}|v|^{m}$ for some $m \geq 0$.

(A6) $\left|\nabla_{x} g(x, t, v)\right| \leq C|v|$.

(A7) $\left|g_{t}(x, t, v)\right| \leq C|v|$.

(A8) Either (a) $1<p<2^{*} / 2, m>0$, (b) $p+p / m<2^{*}, m>0$, or (c) $1<p<2^{*}-1$, where $p$ is given by (A1), and $g$ is either linear in $v$ or does not depend on $v$. Note that the range of exponents $(p, m)$ given by $(\mathrm{A} 8)(\mathrm{c})$ is strictly included in the set $\left(1,2^{*}-1\right) \times$ $\{0,1\}$.

(A9) $\Omega$ is convex such that $\Omega \cap B(z, r)$ are open, bounded, connected domains with Lipschitz boundary for any $z \in \Omega, r>0$ (regular domains in the sense of Sobolev's embedding theorem).

The constants $C, C_{1}, C_{2}$ denote nonnegative numbers which may change from line to line.

The above assumptions are generalizations of those satisfied by the much studied differential equation

$$
u_{t t}-\Delta u \pm u|u|^{p-1} \pm u|u|^{q-1}+a(x, t) u_{t}\left|u_{t}\right|^{m-1}=0
$$

where $1<q<p<2^{*}-1, m \geq 0$, and $a(x, t) \geq 0$. The "-" sign in the above equation corresponds to a situation when energy is introduced into the system, whereas the "+" sign gives us a model whose energy is dissipating. Special cases of this equation arise in quantum field theory (e.g., the Klein-Gordon equation) and in mechanical applications (the dynamics of a membrane in presence of friction when energy is introduced in the system).

1.2. Relationship to previous literature. The interaction between the damping and the source terms has been extensively studied, and pertinent results and conjectures abound in the literature. Thus, it is well-known that 
the source term $f(x, t, u)$ alone can drive the solution to blow up in finite time if it corresponds to accretion of energy [7, 8, 12, 15]. In contrast, the damping term $g\left(x, t, u_{t}\right)$ leads to global existence of weak solutions. The decisive factor in establishing long time behavior of solutions under the interaction of these two effects appears to be the power of the nonlinearity: a higher power of damping would yield long time existence, while a dominant source term will cause the solutions to go to infinity in finite time. This relationship has been studied on both bounded and unbounded domains $[2,3,4,6,9,11,13,16,19]$.

The largest set of exponents $p$ and $m$ for which local existence of solutions was proven for $\mathbb{R}^{n}$ was found in [13] to be given by (A8). This result extended the set of exponents found by J. Serrin, G. Todorova and E. Vitillaro in [16] while using a natural approach with finite energy spaces which are adapted to the equation. For bounded domains we prove that the local existence holds for the same range of exponents as for the entire space, thus generalizing the results of $[6,11]$ where the source term is locally Lipschitz from $H^{1}$ into $L^{2}$. When the mapping $f$ is locally Lipschitz (in other words, $f$ has subcritical exponent $p \leq 3$ for $n=3$ ), local existence of solutions can be proved through monotone semigroup theory (see the Appendix of [4]); for global existence the source term has to additionally satisfy a coercivitytype condition involving the first eigenvalue of the Laplacian. Under these Lipschitz assumptions for the interior source terms the authors of $[3,9,4]$ study existence of solutions and stability issues, where nonlinear source and damping terms act on the boundary of the domain. This setup is different from ours since problems with nonlinear damping on the boundary in general do not satisfy the Lopatinski condition.

We would like to point out that in contrast with the existing literature $([2,6,16])$, our arguments do not make use of the smoothing effect of the damping. This allows us to handle exponents $p$ that go all the way up to $2^{*}-1$ in the case of linear damping and in the case of no damping. Several works mention that the damping is essential in dealing with source terms; our results, however, prove the contrary. For local existence of solutions, at least for subcritical exponents for the source term, the extra regularity given by the damping is not needed. This fact strongly suggests that one should be able to extend the allowable range of exponents to the full box $1<p<2^{*}-1, m \geq 0$, as one naturally expects from the Sobolev embedding theorem. In other words, we conjecture that the exponents $p$ and $m$ do not have to satisfy the restriction

$$
p+p / m<2^{*} \quad \text { for } 2^{*} / 2<p<2^{*}-1 .
$$

The methods used in the study of nonlinear wave equations are usually suited to either bounded or unbounded domains and very few methods are 


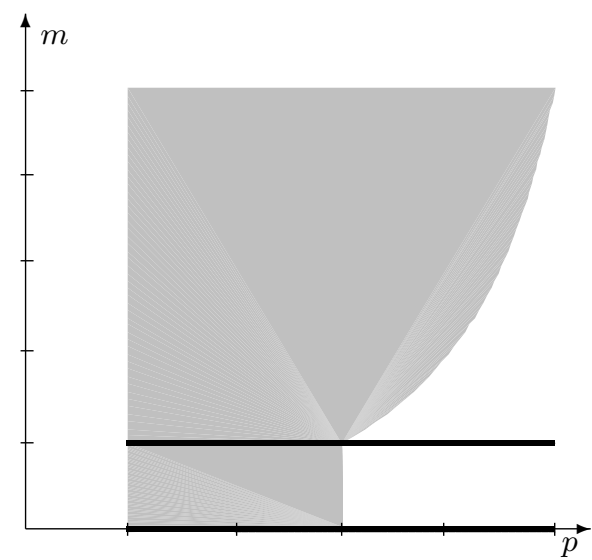

Fig. 1. The range of exponents $p$ and $m$ in $n=3$ where the thick lines mark only the case of linear damping and no damping, so they do not cover nonlinear terms $g(x, t, v)$ whose growth exponent is $m=0$ or $m=1$

applicable in both setups. We show here that our arguments are some of these fortunate situations. The proof relies on two main ingredients: the potential well method due to Sattinger $([12,14])$ and a "patching" argument which was originally used by Crandall and Tartar (see [17, 18]) to show global existence of arbitrarily large solutions for the Broadwell model. In both problems ([13] and [17]) the boundary conditions were absent since the PDEs were considered on the entire space, but we show here that the methods work in the presence of boundary conditions with appropriate modifications. We will often make reference to various results and arguments of [13] without including the proof due to space limitations.

Another fact that distinguishes this paper from other works is that the existence of solutions is proved by passing to the limit for a sequence of approximating solutions where only the source term is approximated with Lipschitz functions, and not the damping term. This allows us to stay within the natural framework of Lebesgue spaces. Note that the usual approach of approximating the damping term with Lipschitz functions is not feasible in Lebesgue spaces (in [16] this obstacle was overcome through a recourse to Orlicz spaces). This is due to the incompatibility between the Lipschitz assumptions and the polynomial growth conditions $v g(x, t, v) \geq$ $(1 / C)|v|^{m},|g(x, t, v)| \leq C|v|^{m-1}$ for some $m \geq 1$, and $g(x, t, 0)=0$, which makes it impossible to work in a Lebesgue space (see [16] for more details).

In conclusion, we summarize the main contributions of this article:

- We provide an extension of the allowed range of exponents $p$ and $m$ through a stronger and more substantial use of the monotonicity of $g$. Prior to this work local existence results for wave equations with in- 
terior source and damping terms on bounded domains were proved only for the range of exponents described by (A8)(a) which corresponds to the case of $f$ Lipschitz from $H^{1}$ into $L^{2}$. Our results extend the prior range in two directions, by allowing (A8)(b) $\left(p+p / m<2^{*}\right)$ and (A8)(c) $\left(p^{*} / 2 \leq p<2^{*}-1\right.$ for linear or no damping).

- In the presence of absorption terms (when $f$ satisfies (A2)*) and damping terms whose range of exponents satisfies (A8) we prove global existence of solutions.

- These results hold for finite energy initial data, not necessarily with compact support as is assumed in other works (e.g. [16, 19]).

- Our work has the advantage of allowing some dependence on $t$ in the function $f$.

- The results apply to wave equations with variable coefficients, as pointed out in Remark 1 of [13].

- This work together with [13] illustrates the great applicability of these methods since they work for problems which are set on bounded and unbounded domains.

2. Main result. We are concerned in this paper with existence of weak solutions, whose definition we present below.

Definition 2.1. Let $\Omega_{T}:=\Omega \times(0, T)$, where $\Omega \subset \mathbb{R}^{n}$ is an open connected set with smooth boundary $\partial \Omega$. Suppose the functions $f$ and $g$ satisfy assumptions (A1) and (A5), and that $u_{0} \in H_{0}^{1}(\Omega) \cap L^{p+1}(\Omega)$ and $u_{1} \in L^{2}(\Omega) \cap L^{m+1}(\Omega)$.

A weak solution on $\Omega_{T}$ of the boundary value problem

$(\mathrm{SW})$

$$
\left\{\begin{array}{l}
u_{t t}-\Delta u+f(x, t, u)+g\left(x, t, u_{t}\right)=0 \text { in } \Omega_{T} ; \\
\left.\left(u, u_{t}\right)\right|_{t=0}=\left(u_{0}, u_{1}\right) ; \\
u=0 \text { on } \partial \Omega \times(0, T)
\end{array}\right.
$$

is any function $u$ satisfying

$$
u \in C\left(0, T ; H_{0}^{1}(\Omega)\right) \cap L^{p+1}\left(\Omega_{T}\right), \quad u_{t} \in L^{2}\left(\Omega_{T}\right) \cap L^{m+1}\left(\Omega_{T}\right),
$$

and

$$
\begin{aligned}
& \int_{\Omega_{T}}\left(u(x, s) \phi_{t t}(x, s)+\nabla u(x, s) \cdot \nabla \phi(x, s)+f(x, s, u) \phi(x, s)\right. \\
& \left.\quad+g\left(x, s, u_{t}\right) \phi(x, s)\right) d x d s=\int_{\Omega}\left(u_{1}(x) \phi(x, 0)-u_{0}(x) \phi_{t}(x, 0)\right) d x
\end{aligned}
$$

for every $\phi \in C_{c}^{\infty}(\Omega \times(-\infty, T))$.

In the following, $|\cdot|_{q}$ is the norm in $L^{q}(\Omega)$. If the domain over which the norm is considered is not clear from the context, then we use $|\cdot|_{q, \Omega}$. 
Below we present our main result and also the main steps of the proof. The steps which are identical with those on the entire $\mathbb{R}^{n}$ (as presented in [13]) are only sketched so that we can focus instead on the patching argument where we have to deal with boundary conditions.

Theorem 2.2 (Main Theorem). Let $\left(u_{0}, u_{1}\right) \in H_{0}^{1}(\Omega) \cap L^{p+1}(\Omega) \times$ $L^{2}(\Omega) \cap L^{m+1}(\Omega)$ and consider the Cauchy problem

$$
\left\{\begin{array}{l}
u_{t t}-\Delta u+f(x, t, u)+g\left(x, t, u_{t}\right)=0 \quad \text { a.e. in } \Omega \times[0, \infty) \\
u(x, t)=0 \quad \text { a.e. }(x, t) \in \partial \Omega \times[0, \infty) \\
\left.\left(u, u_{t}\right)\right|_{t=0}=\left(u_{0}, u_{1}\right) \quad \text { a.e. } x \in \Omega
\end{array}\right.
$$

where $f$ and $g$ satisfy (A0)-(A8). Additionally, assume that $G\left(x, 0, u_{1}\right) \in$ $L^{1}(\Omega)$, where $G(x, t, v)=\int_{0}^{v} g(x, t, u) d u$. Then there exists a time $0<T<1$ such that (SW) admits a weak solution on $[0, T]$ in the sense of Definition 2.1. In addition, if (A2)* is satisfied, then the solution is global, so $T$ can be taken arbitrary. The solution is also continuous in time in the topology of the finite energy space, i.e.

$$
u \in C\left(0, T ; H_{0}^{1}(\Omega)\right) \cap C^{1}\left(0, T ; L^{2}(\Omega)\right) .
$$

REMARK. The restriction $T<1$ is arbitrary, the time of existence is naturally limited by the physics of the problem, since one expects blow-up of solutions whenever the source term dominates the damping.

Proof. In order to facilitate the exposition we present a brief outline of the proof. As mentioned in the introduction, we will approximate the problem with problems that have Lipschitz source terms. We will then use a compactness and monotonicity argument in order to pass to the limit in the sequence of approximating solutions. This approach will actually give us existence of "small" solutions, which we will "patch" to obtain a solution on the entire domain $\Omega$.

STEP 1. We begin by considering the problem with a globally Lipschitz source term in the $u$ argument and arbitrary damping. It is known that for the boundary value problem with $f$ Lipschitz in the third argument and $g$ taken as general as possible under the assumptions $\mathrm{A}_{g}$, we have the following theorem on global existence, uniqueness and regularity of strong solutions for (SW) (see [1] or [13]).

TheOREM 2.3 (Existence and uniqueness of solutions for dissipative wave equations with Lipschitz source terms). Let $\Omega \subset \mathbb{R}^{n}$ be a bounded domain with smooth boundary $\partial \Omega$, and suppose the functions $f(x, t, u)$ and $g(x, t, v)$ satisfy assumptions (A0), (A2), (A3)-(A7), and $f$ is globally Lipschitz in the last argument with Lipschitz constant L, i.e.

$$
|f(x, t, u)-f(x, t, v)| \leq L|u-v|
$$


for every $u, v \in \mathbb{R}$. Let $u_{0}, u_{1} \in H_{0}^{1}(\Omega) \times L^{2}(\Omega)$ with $G\left(x, 0, u_{1}\right) \in L^{1}(\Omega)$, where $G$ is defined by the formula

$$
G(x, t, v)=\int_{0}^{v} g(x, t, y) d y .
$$

Then (SW) admits a unique solution $u$ on the time interval $[0, T]$ such that

$$
u \in C\left(0, T ; H_{0}^{1}(\Omega)\right) \cap L^{p+1}\left(\Omega_{T}\right), \quad u_{t} \in L^{2}\left(\Omega_{T}\right) \cap L^{m+1}\left(\Omega_{T}\right) .
$$

The solutions to the wave equation with Lipschitz source terms and polynomial-like damping have finite speed of propagation. In [13] two statements are proven, one on the growth of the support of solutions, and the second which regards uniqueness of solutions which start from the same initial data. Due to the presence of the nonlinearities $f$ and $g$, these properties are not equivalent, even though the proofs are very similar. The statement concerning the size of the support was proven prior to [13] only for source terms of "good" sign (when their contribution to the energy was a nonnegative quantity). The complete statement of the theorem follows below with the only modification that the balls are permitted to intersect the boundary. The proof remains the same as in [13] but we include it here for the sake of completeness.

TheOREM 2.4 (Finite speed of propagation). Consider the problem (SW) under the hypothesis of Theorem 2.3 (i.e. we additionally require that the source terms are globally Lipschitz). Then

(1) if the initial data $u_{0}, u_{1}$ are compactly supported inside the domain $B\left(x_{0}, R\right) \cap \Omega$, then $u(x, t)=0$ outside $B\left(x_{0}, R+t\right) \cap \Omega$;

(2) if $\left(u_{0}, u_{1}\right),\left(v_{0}, v_{1}\right)$ are two pairs of initial data with compact support, with the corresponding solutions $u(x, t), v(x, t)$, and

$$
\left(u_{0}(x), u_{1}(x)\right)=\left(v_{0}(x), v_{1}(x)\right) \quad \text { for } x \in B\left(x_{0}, R\right) \cap \Omega,
$$

then $u(x, t)=v(x, t)$ in $B\left(x_{0}, R-t\right) \cap \Omega$ for any $t<R$.

Proof. (1) The proof extends an argument used for the linear wave equation by L. Tartar [18].

Assume for now that $f(x, t, u)=0$ for $\left|x-x_{0}\right| \geq R+t$. Since the equation is invariant under translations, without loss of generality we can take $x_{0}=0$. First we approximate the initial data uniformly by smooth functions $\left(u_{0 \eta}, u_{1 \eta}\right)$ with compact support inside $B\left(R_{\eta}\right)$, with $R_{\eta} \nearrow R$ as $\eta \rightarrow 0$. By Theorem 2.3, for any $T>0$, the solution of

$$
\left(\mathrm{SW}_{\eta}\right) \quad\left\{\begin{array}{l}
u_{\eta t t}-\Delta u_{\eta}+f\left(x, t, u_{\eta}\right)+g\left(x, t, u_{\eta t}\right)=0 \quad \text { in } \Omega \times(0, T) ; \\
\left.\left(u_{\eta}, u_{\eta t}\right)\right|_{t=0}=\left(u_{0 \eta}, u_{1 \eta}\right) ; \\
u_{\eta}=0 \quad \text { on } \partial \Omega \times(0, T),
\end{array}\right.
$$


exists on $[0, T]$ and it has the additional regularity

$$
u \in C^{1}\left([0, T] ; L^{2}(\Omega)\right) \quad \text { with } \quad u_{t t}, \Delta u \in L^{2}\left(0, T ; L^{2}(\Omega)\right) .
$$

Consider a function $\phi_{\eta}$ with $\phi_{\eta}(r)=0$ on $\left(-\infty, R_{\eta}\right], \phi_{\eta}(r)>0$ on $\left(R_{\eta}, \infty\right)$, such that $\phi_{\eta}^{\prime}(r) \geq 0$ on $\mathbb{R}$. Since $u_{\eta t} \in L^{\infty}\left(0, T ; H_{0}^{1}\left(B\left(R_{\eta}\right)\right)\right)$, we are allowed to multiply $\left(\mathrm{SW}_{\eta}\right)$ by $u_{\eta t}(t, x) \phi_{\eta}(|x|-t)$, for any $0<t<T$. The quantity

$$
I_{\eta}(t):=\int_{\Omega}\left(\left|u_{\eta t}(x, t)\right|^{2}+\left|\nabla u_{\eta}(x, t)\right|^{2}\right) \phi_{\eta}(|x|-t) d x
$$

is well defined and assume for now that $d I_{\eta} / d t \leq 0$. Then $I_{\eta}(0)=0$, since

$$
\begin{aligned}
I_{\eta}(0)= & \int_{B\left(R_{\eta}\right) \cap \Omega}\left(\left|u_{1 \eta}(x)\right|^{2}+\left|\nabla u_{0 \eta}(x)\right|^{2}\right) \phi_{\eta}(|x|) d x \\
& +\int_{\Omega \backslash B\left(R_{\eta}\right)}\left(\left|u_{1 \eta}(x)\right|^{2}+\left|\nabla u_{0 \eta}(x)\right|^{2}\right) \phi_{\eta}(|x|) d x .
\end{aligned}
$$

The first integral is 0 since $\phi_{\eta}(|x|)=0$ for $|x|<R_{\eta}$. The initial data has support inside the ball $|x|<R_{\eta}$, so the second integral is also zero.

The assumption that the mapping $t \mapsto I_{\eta}(t)$ is decreasing leads us to $I_{\eta}(t) \leq I_{\eta}(0)=0$, which means that $u_{\eta}(x, t)=0$ if $|x|-t>R_{\eta}$. We pass to the limit in $\eta$ (see Theorem 2.2 in [13] on the convergence of approximations of solutions) to obtain $u(x, t)=0$ for $|x|-t>R$, and this is what we set out to prove in part (1).

It then suffices to prove that $d I_{\eta} / d t \leq 0$. For the regularized initial data we have $u_{\eta} \in L^{\infty}\left(0, T ; H_{0}^{1}\left(B\left(R_{\eta}\right)\right)\right), u_{\eta t} \in L^{\infty}\left(0, T ; H_{0}^{1}\left(B\left(R_{\eta}\right)\right)\right), u_{\eta t t} \in$ $L^{1}\left(0, T ; L^{2}\left(B\left(R_{\eta}\right)\right)\right)$, which enables us to compute (we drop the subscript $\eta$ in the remainder of the proof)

$$
\begin{aligned}
& \frac{d I}{d t}(t) \\
& =\int_{\Omega} 2 \phi(|x|-t)\left(u_{t} u_{t t}+\sum_{i=1}^{n} u_{x_{i}} u_{t x_{i}}\right)(x, t) d x-\int_{\Omega} \phi^{\prime}(|x|-t)\left(u_{t}^{2}+|\nabla u|^{2}\right)(x, t) d x \\
& =\int_{\Omega} 2 \phi(|x|-t)\left(u_{t} u_{t t}\right)(x, t) d x-\int_{\Omega} \sum_{i=1}^{n}\left(\left(2 \phi(|x|-t) u_{x_{i}}\right)_{x_{i}} u_{t}\right)(x, t) d x \\
& \quad-\int_{\Omega} \phi^{\prime}(|x|-t)\left(u_{t}^{2}+|\nabla u|^{2}\right)(x, t) d x \\
& =\int_{\Omega} 2 \phi(|x|-t)\left(\left(u_{t t}-\Delta u\right) u_{t}\right)(x, t) d x-\int_{\Omega} \sum_{i=1}^{n} 2 \phi^{\prime}(|x|-t) \frac{x_{i}}{r}\left(u_{x_{i}} u_{t}\right)(x, t) d x \\
& \quad-\int_{\Omega} \phi^{\prime}(|x|-t)\left(u_{t}^{2}+|\nabla u|^{2}\right)(x, t) d x .
\end{aligned}
$$


By (SW),

$$
u_{t t}-\Delta u=-f(x, t, u)-g\left(x, t, u_{t}\right),
$$

hence the assumptions on the support of $\phi$ and $f$, together with the fact that $g$ is nondecreasing, make the first term on the right hand side of the above (multiline) equality negative. We factor out $\phi^{\prime}(|x|-t)$ in the other two terms, and since $\phi^{\prime}(r) \geq 0$ for every $r$, it is enough to show that

$$
u_{t}^{2}+|\nabla u|^{2}+2 \sum_{i=1}^{n} \frac{x_{i}}{|x|} u_{x_{i}} u_{t} \geq 0,
$$

which is obtained by summing the inequalities

$$
\left(\frac{x_{i}}{|x|} u_{t}+u_{x_{i}}\right)^{2} \geq 0
$$

for all $i=1, \ldots, n$.

It remains to show that the function $f$ vanishes for $|x| \geq R+t$. A fixed point argument will establish this fact now. Consider the iterative equation:

$$
\left\{\begin{array}{l}
u_{t t}^{k+1}-\Delta u^{k+1}+f\left(x, t, u^{k}\right)+g\left(x, t, u_{t}^{k+1}\right)=0, \\
\left.\left(u^{k+1}, u_{t}^{k+1}\right)\right|_{t=0}=\left(u_{0}, u_{1}\right) m \\
u^{k+1}=0 \quad \text { on } \partial \Omega \times(0, T),
\end{array}\right.
$$

for every $k \in \mathbb{N}$, with $\left(u^{0}, u_{t}^{0}\right)=\left(u_{0}, u_{1}\right)$. The existence of a unique weak solution is guaranteed by Theorem 2.3. An induction argument, together with the first part of the proof, will show that $u^{k}(x, t)=0$ for $|x|>R+t$ and all $k \in \mathbb{N}$. It is then enough to show that $u^{k}(x, t) \rightarrow u(x, t)$ a.e. as $k \rightarrow \infty$. Since $f$ is Lipschitz we find that $f\left(x, t, u^{k}(x, t)\right)$, which is zero for $|x| \geq R+t$, converges a.e. to $f(x, t, u(x, t))$, hence $f$ vanishes outside the cone $|x|<R+t$. The sequence of difference functions

$$
v^{k}(x, t):=u^{k}(x, t)-u(x, t)
$$

satisfies

$$
\left\{\begin{array}{c}
v_{t t}^{k+1}-\Delta v^{k+1}+f\left(x, t, v^{k}+u\right)-f(x, t, u) \\
\quad+g\left(x, t, v_{t}^{k+1}+u_{t}\right)-g\left(x, t, u_{t}\right)=0 \\
\left.\left(v^{k+1}, v_{t}^{k+1}\right)\right|_{t=0}=(0,0) \\
v^{k+1}=0 \quad \text { on } \partial \Omega \times(0, T) .
\end{array}\right.
$$

Upon multiplication by $v_{t}^{k+1}$ and integration over $(0, t) \times \Omega$, we use the monotonicity of $g$ to derive the following inequality:

$$
\begin{aligned}
\int_{\Omega}\left[\left(v_{t}^{k+1}(x, t)\right)^{2}+\right. & \left.\left|\nabla v^{k+1}(x, t)\right|^{2}\right] d x \\
& \leq \int_{0}^{t} \int_{\Omega} 2\left|f\left(x, t, v^{k}+u\right)-f(x, t, u)\right|\left|v_{t}^{k+1}(x, s)\right| d x d s,
\end{aligned}
$$


which by the Lipschitz assumption on $f$ is

$$
\leq \int_{0}^{t} 2 L\left|v^{k}(s)\right|_{2}\left|v_{t}^{k+1}(s)\right|_{2} d s \leq L \int_{0}^{t}\left[\left|v^{k}(s)\right|_{2}^{2}+\left|v_{t}^{k+1}(s)\right|_{2}^{2}\right] d s .
$$

We now need a bound for $\int_{0}^{t}\left|v^{k}(s)\right|_{2}^{2} d s$, which we obtain by writing

$$
\begin{aligned}
\left|v^{k}(t)\right|_{2}^{2} & =2 \int_{0}^{t} \int_{\Omega} v^{k}(x, s) v_{t}^{k}(x, s) d x d s \\
& \leq \int_{0}^{t} \int_{\Omega}\left[\left(v^{k}(x, s)\right)^{2}+\left(v_{t}^{k}(x, s)\right)^{2}\right] d x d s .
\end{aligned}
$$

Gronwall's inequality for the function $\left|v^{k}(s)\right|_{2}^{2}$ will give us for any $t<T$ the bound

$$
\left|v^{k}(t)\right|_{2}^{2} \leq e^{T} \int_{0}^{t}\left|v_{t}^{k}(s)\right|_{2}^{2} d s .
$$

At this point, to simplify the writing let

$$
\phi^{k}(t):=\int_{0}^{t}\left[\left|v_{t}^{k}(s)\right|_{2}^{2}+\left|\nabla v^{k}(t)\right|_{2}^{2}\right] d s .
$$

By summarizing the estimates above, we see that $\phi^{k+1}$ satisfies the inequality

$$
\phi_{t}^{k+1}(t) \leq L \phi^{k+1}(t)+C \phi^{k}(t),
$$

which after integration becomes

$$
\phi^{k+1}(t) \leq C \int_{0}^{t} e^{L(t-s)} \phi^{k}(s) d s \leq C e^{L T} \int_{0}^{t} \phi^{k}(s) d s .
$$

A simple induction argument shows that

$$
\phi^{k+1}(t) \leq K \frac{C^{k+1} e^{L T(k+1)} t^{k+1}}{(k+1) !},
$$

where $K$ is a bound on $\left|\phi^{1}(t)\right|$ for all $t$ in $[0, T]$. Thus we proved the convergence of $u^{k}(x, t)$ for a.e. $(x, t)$, so $u(x, t)=0$ outside the domain of dependence, i.e. for $x \in \Omega$ with $|x| \geq R+t$. Recall that this is actually proven for the sequence of approximate solutions $u_{\eta}$. By Theorem 2.2 in [13] we have the convergence $u_{\eta}(t) \rightarrow u(t) \in H_{0}^{1}(\Omega)$, hence $u_{\eta}(t) \rightarrow u(t)$ a.e. This concludes the proof of part (1).

(2) We follow here a similar argument to that in part (1). Initially, we work under the assumption that

$$
f(x, t, u(x, t))=f(x, t, v(x, t))
$$


for $|x|<R-t$ (again, take $x_{0}=0$ ). The difference $u-v$ satisfies

$$
\left\{\begin{array}{l}
(u-v)_{t t}-\Delta(u-v)+f(x, t, u)-f(x, t, v)+g\left(x, t, u_{t}\right)-g\left(x, t, v_{t}\right)=0 \\
\left.((u-v),(u-v) t)\right|_{t=0}=(0,0) \\
u-v=0 \text { on } \partial \Omega \times(0, T) .
\end{array}\right.
$$

Consider a function $\psi$, strictly positive on $(-\infty, R)$, such that $\psi(r)=0$ on $[R, \infty)$ and $\psi^{\prime}(r) \leq 0$ everywhere. Define

$$
J(t):=\int_{\mathbb{R}^{n}}\left(\left(u_{t}(x, t)-v_{t}(x, t)\right)^{2}+|\nabla(u(x, t)-v(x, t))|^{2}\right) \psi(|x|-t) d x .
$$

If we show that $d J / d t \leq 0$, then as before, this will imply that $u(x, t)=$ $v(x, t)$ on the support of $\psi(|x|+t)$, i.e. if $|x|<R-t$. The proof follows the same lines as in part (1).

The energy identity allows us to obtain bounds for solutions by using a natural approach based on the physics of the problem (a complete proof can be found in [13]).

Proposition 2.5 (The energy identity). If $u$ is a weak solution of (SW), then under the assumptions of Theorem 2.3 we have the following equality:

$$
\begin{aligned}
E(t)+\int_{\Omega} F(x, t, u(x, t)) & d x-\int_{0}^{t} \int_{\Omega} f_{t}(x, s, u(x, s)) d x d s \\
& +\int_{0}^{t} \int_{\Omega} g\left(x, s, u_{t}(x, s)\right) u_{t}(x, s) d x d s=E(0),
\end{aligned}
$$

where $E(t):=\frac{1}{2}\left|u_{t}(t)\right|_{2}^{2}+\frac{1}{2}|\nabla u(t)|_{2}^{2}$.

At this point we employ the well known Sattinger potential well method to obtain bounds for $|\nabla u(t)|_{2}$ if the initial data satisfy the smallness assumption

$$
\left|\nabla u_{0}\right|_{2}<\alpha, \quad \frac{1}{2}\left|u_{1}\right|_{2}^{2}+\frac{1}{2}\left|\nabla u_{0}\right|_{2}^{2}+\int_{\Omega} F\left(x, 0, u_{0}(x)\right) d x+K|\Omega|<\Phi(\alpha),
$$

where $|\Omega|$ is the Lebesgue measure of $\Omega, K$ is the constant from (A2), and $\Phi$ is the potential well function with $\alpha$ being its global maximum point. More precisely,

$$
\Phi(x)=\frac{x^{2}}{2}-A x^{p}-B x^{q}, \quad x \geq 0,
$$

where $A$ and $B$ are given by

$$
\begin{aligned}
& A=m_{1} C^{p}(R+1)^{n\left(2^{*}-p\right) / 2^{*}}, \\
& B=m_{2} C^{q}(R+1)^{n\left(2^{*}-q\right) / 2^{*}} .
\end{aligned}
$$


and $\alpha$ is the only positive root of the equation $p A \alpha^{p-2}+q B \alpha^{q-2}=1$ (see (3.2) in [13] for more details). As a consequence of Sattinger's potential well argument we obtain

$$
|\nabla u(t)|_{2} \leq \alpha
$$

for all $t>0$ if the smallness assumptions (2.4) are satisfied. If (A2)* is satisfied then the positivity of $F(u)=\int_{0}^{u} f(v) d v$ immediately gives us the desired bounds and we also have global in time estimates for the gradient of the solution. It is important to remark that once the Lipschitz approximations $f_{\varepsilon}$ of $f$ are used in the above problem, these bounds remain independent of $\varepsilon$ so one can use a compactness argument in order to pass to the limit.

STEP 2. At this stage we show how one can construct small solutions which will be later "patched" to obtain a solution on the entire domain. Consider initial data $\left(u_{0}, u_{1}\right)$ such that $u_{0} \in H^{2}(\Omega), u_{1} \in H^{1}(\Omega)$, and $G\left(x, 0, u_{1}\right) \in L^{1}(\Omega)$ (recall that $G(x, t, v)=\int_{0}^{v} g(x, t, y) d y$ ). The higher differentiability assumptions on the initial data are removed with a standard approximation argument, exactly as in [13]. For now, we keep the Lipschitz assumptions for $f$.

For each point $x_{0} \in \Omega$ we will find a domain $\omega_{x_{0}}$ around it such that the smallness assumptions are satisfied on $\omega_{x_{0}}$. We will distinguish between (a) interior points that are far away from the boundary and (b) points which are close to the boundary.

(a) First fix $x_{0} \in \Omega$ far away from the boundary (this will be made more precise below). As in [13] we find a domain $\omega_{x_{0}}$ small enough, and construct new initial data $\left(u_{0}^{x_{0}}, u_{1}^{x_{0}}\right)$ such that $\left(u_{0}^{x_{0}}, u_{1}^{x_{0}}\right)$ satisfy $(2.4)$ inside $\omega_{x_{0}}$. More precisely, $\omega_{x_{0}}=B\left(x_{0}, \varrho\right)$ where the radius $\varrho<1$ is chosen independent of $x_{0}$ and small enough such that

$$
\begin{aligned}
& \varrho<\left(\frac{\Phi(\alpha)}{4 K \omega_{n}}\right)^{1 / n}, \quad\left|\nabla u_{0}\right|_{2, B\left(x_{0}, \varrho\right)}<\alpha / 2, \quad\left|\nabla u_{0}\right|_{2, B\left(x_{0}, \varrho\right)}^{2} \leq \Phi(\alpha) / 8, \\
& 2\left(C^{*} \omega_{n}\right)^{1 / n}\left(\left|\nabla u_{0}\right|_{2, B\left(x_{0}, \varrho\right)}+\left|u_{0}\right|_{2, B\left(x_{0}, \varrho\right)}\right) \leq \min \{\alpha / 2, \sqrt{\Phi(\alpha)} / 8\}, \\
& 4\left(C^{*}\right)^{2} \omega_{n}^{2^{*}-2 / 2^{*}}\left|u_{0}\right|_{2, B\left(x_{0}, \varrho\right)}^{2} \leq \Phi(\alpha) / 8, \quad \frac{1}{2}\left|u_{1}\right|_{2, B\left(x_{0}, \varrho\right)}^{2} \leq \Phi(\alpha) / 4, \\
& m_{1}\left(C^{*}\right)^{p}\left(\left|\nabla u_{0}\right|_{2, B\left(x_{0}, \varrho\right)}+\left|u_{0}\right|_{2, B\left(x_{0}, \varrho\right)}(2 / \varrho+1)\right)^{p} \leq \Phi(\alpha) / 8, \\
& m_{2}\left(C^{*}\right)^{q}\left(\left|\nabla u_{0}\right|_{2, B\left(x_{0}, \varrho\right)}+\left|u_{0}\right|_{2, B\left(x_{0}, \varrho\right)}(2 / \varrho+1)\right)^{q} \leq \Phi(\alpha) / 8,
\end{aligned}
$$

where $\omega_{n}$ is the volume of the unit ball in $\mathbb{R}^{n}$ and $C^{*}$ is the constant from the Sobolev inequality. It can be easily shown that the above inequalities are satisfied by $\varrho<\left(\Phi(\alpha) / 4 K \omega_{n}\right)^{1 / n}$ such that 


$$
\begin{gathered}
\left|\nabla u_{0}\right|_{2, B\left(x_{0}, \varrho\right)}<\min \left\{\frac{\alpha}{2}, \sqrt{\Phi(\alpha) / 8}, \frac{\alpha}{4\left(C^{*} \omega_{n}\right)^{1 / n}},\right. \\
\left.\frac{1}{2 C^{*}}\left(\frac{\Phi(\alpha)}{8 m_{1}}\right)^{1 / p}, \frac{1}{2 C^{*}}\left(\frac{\Phi(\alpha)}{8 m_{2}}\right)^{1 / q}\right\} \\
\left|u_{0}\right|_{2, B\left(x_{0}, \varrho\right)}<\min \left\{\frac{\alpha}{4\left(C^{*} \omega_{n}\right)^{1 / n}}, \frac{1}{\omega_{n} C^{*}} \sqrt{\frac{\Phi(\alpha)}{32 \omega_{n}^{\left(2^{*}-2\right) 2^{*}}},}\right. \\
\left.\frac{1}{8 C^{*}}\left(\frac{\Phi(\alpha)}{8 m_{1}}\right)^{1 / p}, \frac{1}{8 C^{*}}\left(\frac{\Phi(\alpha)}{8 m_{2}}\right)^{1 / q}\right\}
\end{gathered}
$$

and

$$
\left|u_{1}\right|_{2, B\left(x_{0}, \varrho\right)}^{2} \leq \sqrt{\Phi(\alpha) / 8}
$$

The fact that $\varrho$ can be chosen independently of $x_{0}$ is motivated by the equi-integrability of the functions $u_{0}, \nabla u_{0}, u_{1}$. More precisely, for each of these functions we apply the following result of classical analysis:

If $f \in L^{1}(A)$, with $A$ a measurable set, then for every given $\varepsilon>0$, there exists a $\delta>0$ such that $\int_{E}|f(x)| d x<\varepsilon$ for every measurable set $E \subset A$ of measure less than $\delta$ (see [5]).

Note that $\delta$ in the above result does not depend on $E$, hence $\varrho$ does not vary with $x_{0}$.

In order to apply the results of Step 1 we need to know that $u_{0}$ has zero trace on $\partial \omega_{x_{0}}$, so we multiply it by a smooth cut-off function $\theta$ such that

$$
\theta(x)= \begin{cases}1, & \left|x-x_{0}\right| \leq \varrho / 2 \\ 0, & \left|x-x_{0}\right| \geq \varrho\end{cases}
$$

and

$$
|\theta|_{\infty, B\left(x_{0}, \varrho\right)} \leq 1, \quad|\nabla \theta|_{\infty, B\left(x_{0}, \varrho\right)} \leq 2 / \varrho .
$$

On $\omega_{x_{0}}$ we define

$$
u_{0}^{x_{0}}=\theta u_{0}, \quad u_{1}^{x_{0}}=u_{1},
$$

and let $u^{x_{0}}$ be the solution generated by $\left(u_{0}^{x_{0}}, u_{1}^{x_{0}}\right)$. In order to show that the smallness conditions are satisfied we start with the following estimate:

$$
\left|\nabla u_{0}^{x_{0}}\right|_{2, B\left(x_{0}, \varrho\right)} \leq|\theta|_{\infty, B\left(x_{0}, \varrho\right)}\left|\nabla u_{0}\right|_{2, B\left(x_{0}, \varrho\right)}+|\nabla \theta|_{\infty, B\left(x_{0}, \varrho\right)}\left|u_{0}\right|_{2, B\left(x_{0}, \varrho\right)} .
$$

By (2.11), (2.7), Hölder's inequality, and Sobolev's inequality we conclude that

$$
\begin{aligned}
& \left|\nabla u_{0}^{x_{0}}\right|_{2, B\left(x_{0}, \varrho\right)}<\alpha / 2+\left|B\left(x_{0}, \varrho\right)\right|^{1 / n} \frac{2}{\varrho}\left|u_{0}\right|_{2^{*}, B\left(x_{0}, \varrho\right)} \\
& \quad \leq \alpha / 2+2\left(C^{*} \omega_{n}\right)^{1 / n}\left(\left|\nabla u_{0}\right|_{2, B\left(x_{0}, \varrho\right)}+\left|u_{0}\right|_{2, B\left(x_{0}, \varrho\right)}\right) \stackrel{(2.7)}{\leq} \alpha / 2+\alpha / 2=\alpha .
\end{aligned}
$$


so the first part of (2.4) is satisfied. For the second part, first note that by (2.7) we have

$$
\frac{1}{2}\left|u_{1}^{x_{0}}\right|_{2, B\left(x_{0}, \varrho\right)}^{2}<\Phi(\alpha) / 4,
$$

since $u_{1}=u_{1}^{x_{0}}$ on $B\left(x_{0}, \varrho\right)$. Also with a similar argument we have

$$
\begin{gathered}
\left|\nabla u_{0}^{x_{0}}\right|_{2, B\left(x_{0}, \varrho\right)}^{2} \leq|\theta|_{\infty, B\left(x_{0}, \varrho\right)}^{2}\left|\nabla u_{0}\right|_{2, B\left(x_{0}, \varrho\right)}^{2}+|\nabla \theta|_{\infty, B\left(x_{0}, \varrho\right)}^{2}\left|u_{0}\right|_{2, B\left(x_{0}, \varrho\right)}^{2} \\
<\Phi(\alpha) / 8+4\left(C^{*} \omega_{n}\right)^{2 / n}\left(\left|\nabla u_{0}\right|_{2, B\left(x_{0}, \varrho\right)}+\left|u_{0}\right|_{2, B\left(x_{0}, \varrho\right)}\right)^{2} \stackrel{(2.7)}{\leq} \Phi(\alpha) / 4 .
\end{gathered}
$$

For the third term in the second inequality of (2.4), we use assumption (A1)(a), the Sobolev embedding theorem and the restrictions on $\varrho$ in $(2.7)$ to obtain

$$
\begin{aligned}
\int_{B\left(x_{0}, \varrho\right)} F\left(x, 0, u_{0}^{x_{0}}(x)\right) d x \leq & \int_{B\left(x_{0}, \varrho\right)}\left(m_{1}\left|u_{0}^{x_{0}}(x)\right|^{p}+m_{2}\left|u_{0}^{x_{0}}(x)\right|^{q}\right) d x \\
\leq & m_{1}\left(C^{*}\right)^{p}\left(\left|\nabla u_{0}^{x_{0}}\right|_{B\left(x_{0}, \varrho\right)}+\left|u_{0}^{x_{0}}\right|_{B\left(x_{0}, \varrho\right)}\right)^{p} \\
& +m_{2}\left(C^{*}\right)^{q}\left(\left|\nabla u_{0}^{x_{0}}\right|_{B\left(x_{0}, \varrho\right)}+\left|u_{0}^{x_{0}}\right|_{B\left(x_{0}, \varrho\right)}\right)^{q} \\
\leq & m_{1}\left(C^{*}\right)^{p}\left(\left|\nabla u_{0}\right|_{B\left(x_{0}, \varrho\right)}+\left|u_{0}\right|_{B\left(x_{0}, \varrho\right)}(2 / \varrho+1)\right)^{p} \\
& +m_{2}\left(C^{*}\right)^{q}\left(\left|\nabla u_{0}\right|_{B\left(x_{0}, \varrho\right)}+\left|u_{0}\right|_{B\left(x_{0}, \varrho\right)}(2 / \varrho+1)\right)^{q} \\
< & \Phi(\alpha) / 8+\Phi(\alpha) / 8=\Phi(\alpha) / 4 .
\end{aligned}
$$

We also know that $K\left|B\left(x_{0}, \varrho\right)\right|<\Phi(\alpha) / 4$, so by summing the above inequalities we see that $\left(u_{0}^{x_{0}}, u_{1}^{x_{0}}\right)$ satisfies the second inequality of (2.4), i.e.

$$
\begin{aligned}
\frac{1}{2}\left|u_{1}^{x_{0}}\right|_{2, B\left(x_{0}, \varrho\right)}^{2} & +\frac{1}{2}\left|\nabla u_{0}^{x_{0}}\right|_{B\left(x_{0}, \varrho\right)}^{2} \\
& +\int_{B\left(x_{0}, \varrho\right)} F\left(x, 0, u_{0}^{x_{0}}(x)\right) d x+K\left|B\left(x_{0}, \varrho\right)\right|<\Phi(\alpha) .
\end{aligned}
$$

In order to eliminate the higher regularity restrictions on the initial data we approximate $u_{0} \in H^{1}\left(\mathbb{R}^{n}\right), u_{1} \in L^{2}\left(\mathbb{R}^{n}\right)$ by smooth functions and pass to the limit in (2.13) to obtain the conclusions of this step for finite energy initial data.

Since we showed that the pair $\left(u_{0}^{x_{0}}, u_{1}^{x_{0}}\right)$ satisfies (2.4) we apply the results of Step 1 to obtain

$$
\left|\nabla u^{x_{0}}(t)\right|_{2}<\alpha, \quad t>0 .
$$

(b) Assume now that $x_{0} \in \Omega$ is such that $\operatorname{dist}\left(x_{0}, \partial \Omega\right)<\varrho$, where $\varrho$ satisfies (2.7). Let

$$
\omega_{x_{0}}=B\left(x_{0}, \varrho\right) \cap \Omega .
$$

Note that $\partial \omega_{x_{0}}=\gamma_{0} \cup \gamma_{1}$, where $\gamma_{0} \subset \partial \Omega$ and $\gamma_{1} \subset \Omega$. We need to construct $u_{0}^{x_{0}} \in H_{0}^{1}\left(\omega_{x_{0}}\right)$; to this end we use again the cut-off function $\theta$ introduced in part (a). Note that we already have $\left.u_{0}^{x_{0}}\right|_{\gamma_{0}}=0$ so multiplication by $\theta$ 
will ensure that the trace on $\gamma_{1}$ is also zero. The smallness assumptions are satisfied on $\omega_{x_{0}}$ (the proof is identical with the proof in part (a)).

A key observation here is that the geometrical assumption (A9) we imposed on $\partial \Omega$ implies that $\omega_{x_{0}}$ is a nice domain with Lipschitz boundary on which the embedding inequalities (Sobolev and Rellich-Kondrashov) and the Green identities hold (the integration by parts formula is needed to establish the energy identity).

In both cases (a) and (b) we have $u_{0}^{x_{0}}(x)=u_{0}(x)$ and $u_{1}^{x_{0}}(x)=u_{1}(x)$, for all $x \in B\left(x_{0}, \varrho / 2\right) \cap \Omega$.

STEP 3. At this point we construct Lipschitz approximations $f_{\varepsilon}$ for $f$ such that $f_{\varepsilon}$ satisfy the assumptions (A0) with a new function $k_{\varepsilon}$ instead of $k$, and (A1), (A2) with constants $m_{1}, m_{2}, p, q, 1+K$ (see [13] for details). It is easy to see that the coefficients $A, B$ from (2.5), corresponding to $f_{\varepsilon}$, as well as the root $\alpha$, and the radius $\varrho$ chosen above, will not depend on $\varepsilon$. By solving the problem with initial data $\left(u_{0}^{x_{0}}, u_{1}^{x_{0}}\right)$ on $\omega_{x_{0}}$ we obtain a solution $u^{x_{0}}$ which satisfies the estimate

$$
\left|\nabla u_{\varepsilon}^{x_{0}}(t)\right|_{2, \omega_{x_{0}}}<\alpha
$$

for all $t>0$. The maximum time of existence will be restricted through the patching argument by $\varrho / 2$ so all the estimates to follow will be considered for $0 \leq t \leq \varrho / 2$.

The energy identity (2.3), (A2), and the fact that $g$ is increasing imply that

$$
\left|u_{\varepsilon t}^{x_{0}}(t)\right|_{2, \omega_{x_{0}}}^{2}+\left|\nabla u_{\varepsilon}^{x_{0}}(t)\right|_{2, \omega_{x_{0}}}^{2}+\int_{\omega_{x_{0}}} F_{\varepsilon}\left(x, t, u_{\varepsilon}^{x_{0}}(x, t)\right) d x \leq 2 K\left|\omega_{x_{0}}\right|+E(0),
$$

so, with the growth condition (A1)(a) on $F^{\varepsilon}$, Sobolev's inequality and (2.15) we obtain the bound

$$
\begin{aligned}
& \leq 2 K\left|\omega_{x_{0}}\right|+E(0)+\int_{\omega_{x_{0}}}\left(m_{1}\left|u_{\varepsilon}^{x_{0}}(x, s)\right|^{p}+m_{2}\left|u_{\varepsilon}^{x_{0}}(x, s)\right|^{q}\right) d x \\
& \leq 2 K\left|\omega_{x_{0}}\right|+E(0)+C\left(\varrho, m_{1}\right)\left|\nabla u_{\varepsilon}^{x_{0}}(t)\right|_{2, \omega_{x_{0}}}^{p}+C\left(\varrho, m_{2}\right)\left|\nabla u_{\varepsilon}^{x_{0}}(t)\right|_{2, \omega_{x_{0}}}^{q}<C
\end{aligned}
$$

if $0 \leq t \leq \varrho / 2$. By integrating (2.16) over time up to $\varrho / 2$, we deduce from Alaoglu's theorem the existence of a subsequence, denoted also by $u_{\varepsilon}^{x_{0}}$, for which we have the convergences:

$$
\begin{aligned}
& u_{\varepsilon}^{x_{0}} \rightarrow u^{x_{0}} \quad \text { weak }^{*} \text { in } L^{\infty}\left(0, \varrho / 2 ; H_{0}^{1}\left(\omega_{x_{0}}\right)\right), \\
& u_{\varepsilon t}^{x_{0}} \rightarrow u_{t}^{x_{0}} \quad \text { weak }^{*} \text { in } L^{\infty}\left(0, \varrho / 2 ; L^{2}\left(\omega_{x_{0}}\right)\right) .
\end{aligned}
$$

Also, by Aubin's theorem and the Rellich-Kondrashov compact embedding 
theorem we have the convergence

$$
u_{\varepsilon}^{x_{0}} \rightarrow u^{x_{0}} \quad \text { strongly in } L^{2}\left((0, \varrho / 2) \times \omega_{x_{0}}\right),
$$

so for a subsequence we have

$$
u_{\varepsilon}^{x_{0}}(x, t) \rightarrow u^{x_{0}}(x, t) \quad \text { a.e. }(x, t) \in \omega_{x_{0}} \times(0, \varrho / 2) .
$$

It is shown in [13] that this implies

$$
f_{\varepsilon}\left(x, t, u_{\varepsilon}^{x_{0}}(x, t)\right) \rightarrow f\left(x, t, u^{x_{0}}(x, t)\right) \quad \text { a.e. }(x, t) \in \omega_{x_{0}} \times(0, \varrho / 2),
$$

so we finally have (after using the subcritical growth of $f$ )

$$
f_{\varepsilon}\left(x, t, u_{\varepsilon}^{x_{0}}(x, t)\right) \rightarrow f\left(x, t, u^{x_{0}}(x, t)\right) \quad \text { in } L^{1}\left(\omega_{x_{0}} \times(0, \varrho / 2)\right),
$$

hence also in the sense of distributions.

A monotonicity argument will be applied in order to pass to the limit in the nonlinear dissipative term $g\left(x, t, u_{\varepsilon t}^{x_{0}}\right)$. From (A4), the energy identity and the bounds on $F_{\varepsilon}$ obtained above, we have

$$
\begin{aligned}
& \quad\left|u_{\varepsilon t}^{x_{0}}(t)\right|_{2, \omega_{x_{0}}}^{2}+\left|\nabla u_{\varepsilon}^{x_{0}}(t)\right|_{2, \omega_{x_{0}}}^{2}+\int_{0}^{t} \int_{\omega_{x_{0}}}\left|u_{\varepsilon t}^{x_{0}}(x, s)\right|^{m+1} d x d s \\
& \leq\left|u_{\varepsilon t}^{x_{0}}(t)\right|_{2, \omega_{x_{0}}}^{2}+\left|\nabla u_{\varepsilon}^{x_{0}}(t)\right|_{2, \omega_{x_{0}}}^{2}+\int_{0}^{t} \int_{\omega_{x_{0}}} g\left(x, s, u_{\varepsilon t}^{x_{0}}(x, s)\right) u_{\varepsilon t}^{x_{0}}(x, s) d x d s \leq C .
\end{aligned}
$$

Therefore, we can again extract a subsequence $u_{\varepsilon}^{x_{0}}$ such that

$$
\begin{array}{ll}
u_{\varepsilon t}^{x_{0}} \rightarrow u_{t}^{x_{0}} & \text { in } L^{m+1}\left((0, \varrho / 2) \times \omega_{x_{0}}\right), \\
g\left(x, t, u_{\varepsilon t}^{x_{0}}\right) \rightarrow \xi & \text { in } L^{(m+1)^{\prime}}\left((0, \varrho / 2) \times \omega_{x_{0}}\right) .
\end{array}
$$

Passing to the limit in $\varepsilon$ we obtain (we drop the ${ }^{x_{0}}$ superscript for $u$ in what follows)

$$
u_{t t}-\Delta u+f(x, t, u)+\xi=0 \quad \text { in the sense of distributions. }
$$

We need to verify that $\xi=g\left(x, t, u_{t}\right)$.

Let $u_{\varepsilon}, u_{\eta}$ be two terms in the sequence $\left(u_{\varepsilon}\right)_{\varepsilon>0}$. We subtract the equations satisfied by $u_{\varepsilon}, u_{\eta}$ to obtain

$$
\begin{aligned}
& \int_{\omega_{x_{0}}}\left(\left|u_{\varepsilon t}(x, t)-u_{\eta t}(x, t)\right|^{2}+\left|\nabla u_{\varepsilon}(x, t)-\nabla u_{\eta}(x, t)\right|^{2}\right) d x \\
+ & \int_{0}^{t} \int_{\omega_{x_{0}}}\left(g\left(x, s, u_{\varepsilon t}(x, s)\right)-g\left(x, s, u_{\eta t}(x, s)\right)\right)\left(u_{\varepsilon t}(x, s)-u_{\eta t}(x, s)\right) d x d s \\
= & -\int_{0}^{t} \int_{\omega_{x_{0}}}\left(f_{\varepsilon}\left(x, s, u_{\varepsilon}(x, s)\right)-f_{\eta}\left(x, s, u_{\eta}(x, s)\right)\right)\left(u_{\varepsilon t}(x, s)-u_{\eta t}(x, s)\right) d x d s .
\end{aligned}
$$


The standard process through which we obtained the above equality (multiplication by $u_{\varepsilon t}-u_{\eta t}$, integration by parts etc.) is motivated exactly as in obtaining the energy identity for equations with Lipschitz source terms with nonlinear damping (Proposition 2.5).

In the spirit of the monotonicity argument found in [9, p. 518] (although we are here in a different setup with approximations on the source terms, not on the damping) we obtain

$$
\lim _{\eta, \varepsilon \rightarrow 0} \int_{0}^{t} \int_{\omega_{x_{0}}}\left(g\left(x, s, u_{\varepsilon t}\right)-g\left(x, s, u_{\eta t}\right)\right)\left(u_{\varepsilon t}-u_{\eta t}\right) d x d s=0 .
$$

This equality is obtained by passing to the limit as $\eta, \varepsilon \rightarrow 0$ in (2.24). The convergence to zero of the term on the RHS of (2.24) is shown below. After using the positivity of all the terms on the LHS the conclusion of (2.25) follows. We will now repeat the argument used in [13] to show the convergence to zero of the difference of source terms, i.e.

$$
\lim _{\eta, \varepsilon \rightarrow 0} \int_{0}^{t} \int_{\omega_{x_{0}}}\left(f_{\varepsilon}\left(x, s, u_{\varepsilon}\right)-f_{\eta}\left(x, s, u_{\eta}\right)\right)\left(u_{\varepsilon t}-u_{\eta t}\right) d x d s=0 .
$$

First we multiply out the quantities in the integrand and show convergence for each of them. We start with the study of the "non-mixed" product $f_{\varepsilon}\left(u_{\varepsilon}\right) u_{\varepsilon t}$ (identical analysis for $f_{\eta}\left(u_{\eta}\right) u_{\eta t}$ ). We have the equality

$$
\begin{aligned}
& \int_{0}^{t} \int_{\omega_{x_{0}}} f_{\varepsilon}\left(x, s, u_{\varepsilon}(x, s)\right) u_{t \varepsilon}(x, s) d x d s \\
& \quad=\left.\int_{\omega_{x_{0}}} F_{\varepsilon}\left(x, s, u_{\varepsilon}(x, s)\right) d x\right|_{s=0} ^{s=t}-\int_{0}^{t} \int_{\omega_{x_{0}}} F_{\varepsilon t}\left(x, s, u_{\varepsilon}(x, s)\right) d x d s,
\end{aligned}
$$

where we notice that we can pass to the limit in the first term of the RHS by (2.16) combined with the subcritical growth for $F$. For the second term, by (A2), we have $\left|F_{\varepsilon t}\left(u_{\varepsilon}\right)\right| \leq K\left|u_{\varepsilon}\right|$, and since $u_{\varepsilon}$ is bounded in $L^{1}$ (as a consequence of the Sobolev embedding theorem), by the Lebesgue dominated convergence we get $f_{\varepsilon}\left(x, t, u_{\varepsilon}\right) \rightarrow f(x, t, u)$ in $L^{1}\left((0, t) \times B\left(x_{0}, \varrho^{*}\right)\right)$.

The analysis of the "mixed" terms $\left(f_{\varepsilon}\left(u_{\varepsilon}\right) u_{\eta t}\right.$ and $\left.f_{\eta}\left(u_{\eta}\right) u_{\varepsilon t}\right)$ will, however, impose some restrictions on the exponents $p$ and $m$. We first analyze $f_{\varepsilon}\left(u_{\varepsilon}\right) u_{\eta t}$ which converges a.e. to $f(u) u_{\eta t}$ as $\varepsilon \rightarrow 0$ by (2.19). By Egorov's theorem for every $\delta>0$ there exists a set $A \subset(0, t) \times \omega_{x_{0}}$ with $|A|<\delta$ such that $f_{\varepsilon}\left(u_{\varepsilon}\right) u_{\eta t} \rightarrow f(u) u_{\eta t}$ uniformly (hence, in $\left.L^{1}\right)$ on $(0, t) \times \omega_{x_{0}} \backslash A$.

We write

$$
\int_{0}^{t} \int_{\omega_{x_{0}}} f_{\varepsilon}\left(u_{\varepsilon}\right) u_{\eta t} d x d s=\int_{(0, t) \times \omega_{x_{0}} \backslash A} f_{\varepsilon}\left(u_{\varepsilon}\right) u_{\eta t} d x d s+\int_{A} f_{\varepsilon}\left(u_{\varepsilon}\right) u_{\eta t} d x d s .
$$


Due to the uniform convergence $f_{\varepsilon}\left(u_{\varepsilon}\right) u_{\eta t} \rightarrow f(u) u_{\eta t}($ as $\varepsilon \rightarrow 0)$ on $(0, t) \times$ $\omega_{x_{0}} \backslash A$, we have

$$
\lim _{\varepsilon \rightarrow 0} \int_{(0, t) \times \omega_{x_{0}} \backslash A} f_{\varepsilon}\left(u_{\varepsilon}\right) u_{\eta t} d x d s=\int_{(0, t) \times \omega_{x_{0}} \backslash A} f(u) u_{\eta t} d x d s .
$$

In order to analyze the integral on $A$ in (2.27) we apply Hölder's inequality with conjugate exponents $\alpha, \beta$, and $\gamma$ :

$$
\int_{A}\left|f_{\varepsilon}\left(u_{\varepsilon}\right) u_{\eta t}\right| d x d s \leq C\left(\int_{A}\left|u_{\varepsilon}\right|^{\alpha p} d x d s\right)^{1 / \alpha}\left(\int_{A}\left|u_{\eta t}\right|^{\beta} d x d s\right)^{1 / \beta}|A|^{1 / \gamma} .
$$

Our goal is to bound the first two factors on the RHS above, and to this end we have two options for choosing $\alpha, \beta$, and $\gamma$. First we take

$$
\alpha=\frac{2^{*}}{p}, \quad \beta=2, \quad \gamma=\frac{2 \cdot 2^{*}}{2^{*}-2 p},
$$

and by the Sobolev embedding theorem and by (2.15) we have the desired bounds in (2.29) if $\gamma>0$. The positivity of $\gamma$ amounts to $p<2^{*} / 2$, which is condition (a) in (A8). The second choice is

$$
\alpha=\frac{m+1}{m-(m+1) \nu}, \quad \beta=m+1, \quad \gamma=\frac{1}{\nu},
$$

for some $0<\nu<1$. We need to require that $\alpha p \leq 2^{*}$ and by letting $\nu \rightarrow 0(\nu \neq 0)$, we get the restriction $p+p / m<2^{*}$ (condition (b) in (A8)).

Now we go back in (2.27) and take $\lim _{\delta \rightarrow 0} \lim _{\varepsilon \rightarrow 0}$ on both sides. First, in (2.28) take $\lim _{\delta \rightarrow 0}$ and notice that we can bound the integrand as in (2.29), and since $(0, t) \times \omega_{x_{0}} \times(0, \varrho / 2) \backslash A \rightarrow(0, t) \times \omega_{x_{0}} \times(0, \varrho / 2)$ as $\delta \rightarrow 0$, by the Lebesgue dominated convergence theorem we have

$$
\lim _{\delta \rightarrow 0} \lim _{\varepsilon \rightarrow 0} \int_{(0, t) \times \omega_{x_{0} \backslash A}} f_{\varepsilon}\left(u_{\varepsilon}\right) u_{\eta t} d x d s=\int_{(0, t) \times \omega_{x_{0}}} f(u) u_{\eta t} d x d s .
$$

From (2.29) we have

$$
\lim _{\delta \rightarrow 0} \lim _{\varepsilon \rightarrow 0} \int_{A}\left|f_{\varepsilon}\left(u_{\varepsilon}\right) u_{\eta t}\right| d x d s \leq \lim _{\delta \rightarrow 0} C|A|^{1 / \gamma} \lim _{\varepsilon \rightarrow 0} M=0,
$$

where $M$ is a bound for the first two factors on the RHS of (2.29). Thus we obtain

$$
\lim _{\varepsilon \rightarrow 0} \int_{0}^{t} \int_{\omega_{x_{0}}} f_{\varepsilon}\left(u_{\varepsilon}\right) u_{\eta t} d x d s=\int_{0}^{t} \int_{\omega_{x_{0}}} f(u) u_{\eta t} d x d s .
$$

We let $\eta \rightarrow 0$ and by using (2.20) and (2.17) we find that the limit of this mixed term is $\int_{0}^{t} \int_{\omega_{x_{0}}} f(u) u_{t} d x d s$. The analysis of the second mixed term is similar and so we omit it. Thus we conclude that (2.26) holds, hence (2.25) is valid. Note that (2.24)-(2.26) also give us the continuity with respect to 
time,

$$
u \in C\left(0, T ; H_{0}^{1}(\Omega)\right) \cap C^{1}\left(0, T ; L^{2}(\Omega)\right) .
$$

We continue the monotonicity argument by multiplying out the quantities in (2.25). By using (2.17) and (2.22) we have

$$
\begin{aligned}
\lim _{\varepsilon \rightarrow 0} \int_{0}^{t} \int_{\omega_{x_{0}}}\left(g\left(x, s, u_{\varepsilon t}\right) u_{\varepsilon t}-\xi(x, s) u_{\varepsilon t}\right. & \left.-g\left(x, s, u_{\varepsilon t}\right) u_{t}\right) d x d s \\
& +\lim _{\eta \rightarrow 0} \int_{0}^{t} \int_{\omega_{x_{0}}} g\left(x, s, u_{\eta t}\right) u_{\eta t} d x d s=0 .
\end{aligned}
$$

After using again (2.17) and (2.22) and changing $\eta$ to $\varepsilon$ in the last term above we get

$$
2 \lim _{\varepsilon \rightarrow 0} \int_{0}^{t} \int_{\omega_{x_{0}}} g\left(x, s, u_{\varepsilon t}\right) u_{\varepsilon t} d x d s=2 \int_{0}^{t} \int_{\omega_{x_{0}}} \xi(x, s) u_{t} d x d s .
$$

By the monotonicity of $g$ we have

$$
\int_{0}^{t} \int_{\omega_{x_{0}}}\left(g\left(x, s, u_{\varepsilon t}(x, s)\right)-g(x, s, \phi(x, s))\right)\left(u_{\varepsilon t}(x, s)-\phi(x, s)\right) d x d s \geq 0
$$

for every $\phi \in L^{m+1}\left((0, t) \times \omega_{x_{0}}\right)$. In order to close the argument we use (2.30) to obtain

$$
\begin{aligned}
\lim _{\varepsilon \rightarrow 0} \int_{0}^{t} \int_{\omega_{x_{0}}}( & \left.\left(x, s, u_{\varepsilon t}(x, s)\right)-g(x, s, \phi(x, s))\right)\left(u_{\varepsilon t}-\phi(x, s)\right) d x d s \\
\leq & \int_{0}^{t} \int_{\omega_{x_{0}}}(\xi(x, s)-g(x, s, \phi(x, s)))\left(u_{t}(x, s)-\phi(x, s)\right) d x d s
\end{aligned}
$$

By combining (2.31) and (2.30) we obtain

$$
\int_{0}^{t} \int_{\omega_{x_{0}}}(\xi(x, s)-g(x, s, \phi(x, s)))\left(u_{t}(x, s)-\phi(x, s)\right) d x d s \geq 0
$$

for all $t<\varrho / 2$, so by passing to limit as $t \rightarrow \varrho / 2$, it holds also for $t=\varrho / 2$. We choose $\phi$ appropriately $\left(\phi_{ \pm}:=u_{t} \pm \lambda v\right.$ for $\left.\lambda>0\right)$ and take $v$ arbitrary in $C_{c}^{\infty}\left(\omega_{x_{0}}\right)$. Let $\lambda \rightarrow 0$ for both choices, $\phi_{+}$and $\phi_{-}$, to obtain the desired equality $\xi=g\left(u_{t}\right)$.

If $m=0$ and $g$ does not depend on $u_{t}$, or $m=1$ and $g$ is linear in $u_{t}$, then one does not need the monotonicity argument in order to obtain $g\left(u_{t}\right)=\xi$, only $(2.17)_{2}$ and $(2.22)_{1}$. Since there is no other restriction imposed on $p$, these values for $p$ and $m$ cover case (c) in (A8).

At this stage we have global existence of the small solutions $u^{x_{0}}$ on the domains $\omega_{x_{0}}$. Through the patching argument described in Step 4 we will construct an arbitrarily large solution $u$ on the entire domain $\Omega$. 
STEP 4. In this last step will put together the previous arguments to construct the solution $u$ from arbitrarily large initial data $\left(u_{0}, u_{1}\right)$ considered on the domain $\Omega$. The order in which we assemble all the pieces is very important, so for a better understanding of this intricate procedure we identify the following substeps:

Step 4.1. Cut the initial data in small pieces on bounded domains $\omega_{x_{0}}$ and for each piece obtain global existence of solutions for the approximate problems with Lipschitz source terms $f_{\varepsilon}$.

Step 4.2. For each bounded domain, obtain bounds for $\left|\nabla u_{\varepsilon}^{x_{0}}(t)\right|_{2}$ and pass to the limit in the approximate solutions; hence, we obtain existence for the problem with a general source term.

Step 4.3. Up to some time $T<1$, "patch all solutions" obtained in Step 4.2 to obtain a solution for the problem with a general source term with initial data on $\Omega$.

Step 4.4. Show that the solution defined in Step 4.3 is well defined and it is the solution generated by the initial data $\left(u_{0}, u_{1}\right)$.

Below is a detailed discussion of the above construction.

STEP 4.1. Let $d>0$. Consider a lattice of points $x_{k}, k \in \mathbb{N}$, in $\Omega$ situated at distance $d$ from each other, such that in every ball of radius $d$ we find at least one $x_{k}$. With $\varrho$ that satisfies (2.7) (where $\varrho$ depends only on the norms of the initial data), construct the balls of radius $\varrho$ centered at $x_{k}$. The procedure outlined in Step 2 for truncating the initial data around $x_{0}$ to obtain a "small piece" denoted by $\left(u_{0}^{x_{0}}, u_{1}^{x_{0}}\right)$, will now be used to construct around each $x_{k}$ the truncations $\left(u_{0}^{x_{k}}, u_{1}^{x_{k}}\right)$ which will satisfy the smallness assumptions

$$
\begin{aligned}
& \left|\nabla u_{0}^{x_{k}}\right|_{\omega_{x_{k}}}<\alpha, \\
& \frac{1}{2}\left|u_{1}^{x_{k}}\right|_{\omega_{x_{k}}}^{2}+\frac{1}{2}\left|\nabla u_{0}^{x_{k}}\right|_{\omega_{x_{k}}}^{2}+\int_{\omega_{x_{k}}} F\left(x, 0, u_{0}^{x_{k}}(x)\right) d x+K\left|\omega_{x_{k}}\right|<\Phi(\alpha) .
\end{aligned}
$$

On each ball $\omega_{x_{k}}$ we apply Theorem 2.3 to obtain global existence of solutions $u_{\varepsilon}^{x_{k}}$ for the problem (SW) with initial data $\left(u_{0}^{x_{k}}, u_{1}^{x_{k}}\right)$ and with the Lipschitz approximations $f_{\varepsilon}$ for the source term.

STEP 4.2. At this point, the arguments of Step 3 for passing to the limit as $\varepsilon \rightarrow 0$ in the sequence of approximate problems are applied, where $x_{0}$ is successively replaced by different $x_{k}$ 's. First, we apply Sattinger's argument to estimate $\left|\nabla u_{\varepsilon}^{x_{k}}\right|_{2}$ on $\omega_{x_{k}}$ for each $k$. (Note that we need the smallness assumptions of Step 4.1.) The convergence $u_{\varepsilon}^{x_{k}} \rightarrow u^{x_{k}}$ holds on every domain $\omega_{x_{k}} \times(0, \varrho / 2)$, so we obtain a global (in time) solution to the boundary value problem (SW) for $\omega_{x_{k}}$, for every $k$. 
STEP 4.3. The solutions $u^{x_{k}}$ found in Step 4.2 will now be "patched" together to obtain our general solution. First, for $k \in \mathbb{N}$ let

$$
C_{k}:=\left\{(y, s) \in \mathbb{R}^{3} \times[0, \infty):\left|y-x_{k}\right| \leq \varrho / 2-s\right\}
$$

be the backward cone with vertex at $\left(x_{k}, \varrho / 2\right)$. For $d$ small enough (i.e. for $0<d<\varrho / 2$ ) any two neighboring cones $C_{k}$ and $C_{j}$ will intersect. On every intersection set

$$
I_{k, j}:=C_{k} \cap C_{j}
$$

the maximum of the time coordinate is $(\varrho-d) / 2$ (see Figure 3 below).

For $t<\varrho / 2$ we define the piecewise function

$$
u(x, t):=u^{x_{k}}(x, t) \quad \text { if }(x, t) \in C_{k} .
$$

This solution is defined only up to time $(\varrho-d) / 2$, since this is the height of the intersection set of two cones with their vertices situated at distance $d$ from each other. By letting $d \rightarrow 0$ we can obtain a solution well defined up to time $\varrho / 2$. Thus, we have defined $u$ up to time $\varrho / 2$, which is the height of all cones $C_{k}$. Every pair $(x, t) \in \mathbb{R}^{n} \times(0, \varrho / 2)$ belongs to at least one $C_{k}$, so in order to show that the function from (2.33) is well defined, we need to check that it is single-valued on the intersection of two cones. Also, we need to show that it is the solution generated by the initial data $\left(u_{0}, u_{1}\right)$. Both proofs will be done in the next step.

STEP 4.4. To prove the desired properties, we will go back and look at the solutions $u^{x_{k}}$ as limits of the approximate solutions $u_{\varepsilon}^{x_{k}}$.

Consider first balls which do not intersect the boundary.

For each $k \in \mathbb{N}$ we have $\left(u_{0}^{x_{k}}, u_{1}^{x_{k}}\right)=\left(u_{0}, u_{1}\right)$ for all $x \in \omega_{x_{k}}=\left\{y \in \mathbb{R}^{n}\right.$ : $\left.\left|y-x_{k}\right|<\varrho / 2\right\}$ (see the construction of the truncations $\left(u_{0}^{x_{k}}, u_{1}^{x_{k}}\right)$ in Step 2). Therefore, $u_{\varepsilon}^{x_{k}}$ (defined in Step 4.1) is an approximation of the solution generated by the initial data $\left(u_{0}, u_{1}\right)$ on $C_{k}$ (from the uniqueness property given by Theorem 2.4(2)). We let $\varepsilon \rightarrow 0$ (use the argument from Step 3) to show that the solution $u$ on each $C_{k}$ is generated by the initial data $\left(u_{0}, u_{1}\right)$.

To show that $u$ defined by $(2.33)$ is a proper function, we use the same result of uniqueness given by the finite speed of propagation. First note that for $n \geq 3$ the intersection $I_{k, j}$ is not a cone, but it is contained in the cone $C_{k, j}$ with vertex at $\left(\left(x_{k}+x_{j}\right) / 2,(\varrho-d) / 2\right)$ of height $(\varrho-d) / 2$. In this cone we use the uniqueness asserted by the finite speed of propagation as follows. First note that the cone $C_{k, j}$ contains the set $I_{k, j}$, but $C_{k, j} \subset C_{k} \cup C_{j}$. In $C_{k}$ we have the solution $u_{\varepsilon}^{x_{k}}$, while in $C_{j}$ the solution is given by $u_{\varepsilon}^{x_{j}}$ (see construction in 4.1); hence, in $C_{k, j}$ we have two solutions and we need to show they are equal. Since $u_{\varepsilon}^{x_{k}}$ and $u_{\varepsilon}^{x_{j}}$ start from the same initial data $\left(\left(u_{0}^{x_{k}}, u_{1}^{x_{k}}\right)=\left(u_{0}, u_{1}\right)=\left(u_{0}^{x_{j}}, u_{1}^{x_{j}}\right)\right.$ on $\left.\omega_{x_{k}} \cap B_{j}\right)$, they are equal on $C_{k, j}$ 


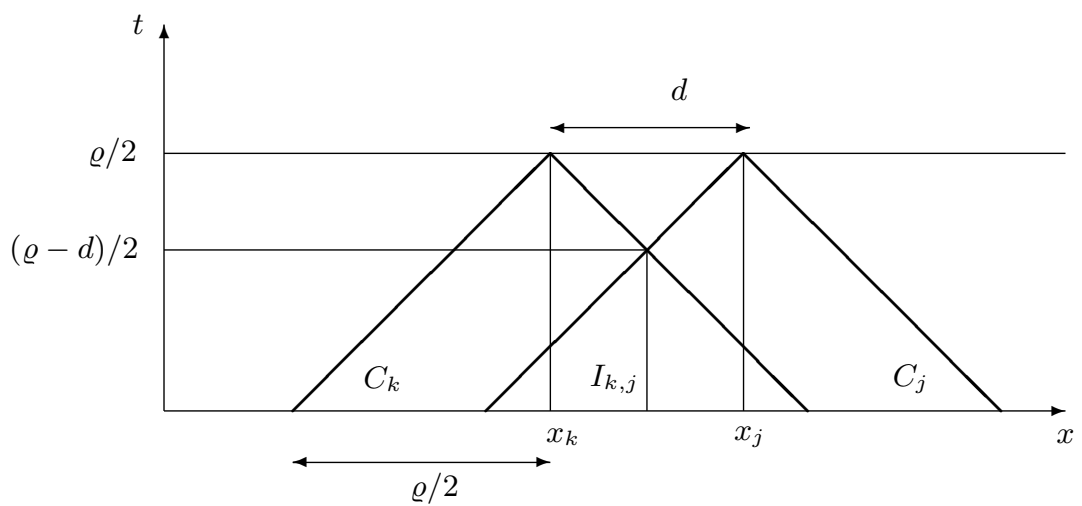

Fig. 2. The intersection of the cones $C_{k}$ and $C_{j}$

by Theorem 2.4(2). We let $\varepsilon \rightarrow 0$ to obtain $u_{k}^{x_{0}}=u_{j}^{x_{0}}$ in $C_{k, j}$, and since $I_{k, j} \subset C_{k, j}$ we have $u_{k}^{x_{0}}=u_{j}^{x_{0}}$ on $I_{k, j}$. Therefore, $u$ is a single-valued (proper) function.

Two issues arise when we treat the case of balls that intersect the boundary. The first is the geometry of the intersection sets, so that we can apply the uniqueness from Theorem 2.4(2). The second is to show that the Dirichlet boundary conditions are still satisfied.

Assume $B\left(x_{j}, \varrho\right)$ and $B\left(x_{k}, \varrho\right)$ intersect $\partial \Omega$ and let $\omega_{x_{j}}:=B\left(x_{j}, \varrho\right) \cap \Omega$ and $\omega_{x_{k}}:=B\left(x_{k}, \varrho\right) \cap \Omega$. On $\omega_{x_{k}}$ and $\omega_{x_{j}}$ we have again two solutions which by the same finite speed of propagation argument can be shown to coincide on the intersection. In the picture below the line $x=0$ represents $\partial \Omega$. We see that on intersecting the boundary $\partial \Omega$ with the cones $C_{k}$ and $C_{j}$ we can still show that on the intersection $I_{k, j}$ we have a unique solution.

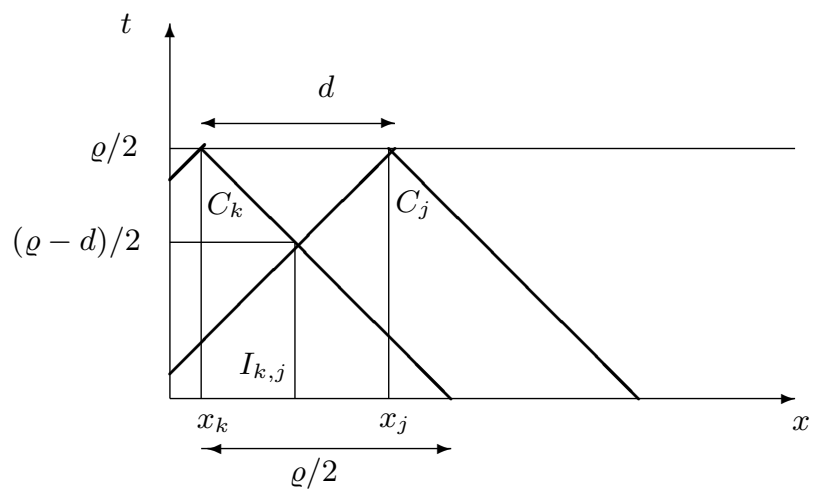

Fig. 3. The intersection of the domains $C_{k} \cap \Omega$ and $C_{j} \cap \Omega$

The above method of using cut-off functions and "patching" solutions based on uniqueness will also work when we additionally assume (A2)*. Since 
we can choose the height of the cones as large as we wish, the solutions exist globally in time under the positivity hypothesis for $F$.

The boundary conditions are satisfied since $u^{x_{k}}=0$ on each $\partial \omega_{x_{k}} \cap \partial \Omega$. Since the solution $u$ is obtained by patching all the solutions $u^{x_{k}}$ we obtain $u=0$ on $\partial \Omega$.

Acknowledgements and errata to [13]. The author would like to warmly thank the referee for pointing out an error in the original draft of the manuscript and for making other suggestions which helped improve the paper. The error (which unfortunately also appeared in [13]) regards the range of exponents $(p, m)$ which does not include the full set $\left(1,2^{*}-1\right) \times$ $\{0,1\}$, as previously thought. In the case $(p, m) \in\left(1,2^{*}-1\right) \times\{0,1\}$ we can only allow $g$ linear in the velocity argument (subcase of $m=1$ ), or $g$ independent of the velocity (subcase of $m=0$ ). The correct assumptions and arguments for dealing with this case are outlined in this paper.

\section{References}

[1] V. Barbu, Nonlinear Semigroups and Differential Equations in Banach Spaces, Editura Academiei, Bucureşti, and Noordhoff, Leyden, 1976.

[2] V. Barbu, I. Lasiecka and M. A. Rammaha, On nonlinear wave equations with degenerate damping and source terms, Trans. Amer. Math. Soc. 357 (2005), 25712611.

[3] M. Cavalcanti, V. Domingos Cavalcanti and I. Lasiecka, Well-posedness and optimal decay rates for the wave equation with nonlinear boundary damping-source interaction, J. Differential Equations 236 (2007), 407-459.

[4] I. Chueshov, M. Eller and I. Lasiecka, On the attractor for a semilinear wave equation with critical exponent and nonlinear boundary dissipation, Comm. Partial Differential Equations 27 (2002), 1901-1951.

[5] L. C. Evans and R. F. Gariepy, Measure Theory and Fine Properties of Functions, Stud. Adv. Math., CRC Press, 1992.

[6] V. Georgiev and G. Todorova, Existence of a solution of the wave equation with nonlinear damping and source terms, J. Differential Equations 109 (1994), 295-308.

[7] R. T. Glassey, Blow-up theorems for nonlinear wave equations, Math. Z. 132 (1973), 183-203.

[8] F. John, Blow-up of solutions of nonlinear wave equations in three space dimensions, Manuscripta Math. 28 (1979), 235-268.

[9] I. Lasiecka and D. Tataru, Uniform boundary stabilization of semilinear wave equations with nonlinear boundary damping, Differential Integral Equations 6 (1993), 507-533.

[10] I. Lasiecka and D. Toundykov, Energy decay rates for the semilinear wave equation with nonlinear localized damping and source terms, Nonlinear Anal. 64 (2006), 17571797.

[11] J.-L. Lions and W. A. Strauss, On some nonlinear evolution equations, Bull. Soc. Math. France 93 (1965), 43-96.

[12] L. E. Payne and D. H. Sattinger, Saddle points and instability of nonlinear hyperbolic equations, Israel J. Math. 22 (1975), 273-303. 
[13] P. Radu, Weak solutions to the Cauchy problem of a semilinear wave equation with damping and source terms, Adv. Differential Equations 10 (2005), 1261-1300.

[14] D. H. Sattinger, On global solution of nonlinear hyperbolic equations, Arch. Ration. Mech. Anal. 30 (1968), 148-172.

[15] J. Schaeffer, The equation $u_{t t}-\Delta u=|u|^{p}$ for the critical value of $p$, Proc. Roy. Soc. Edinburgh Sect. A 101 (1985), 31-44.

[16] J. Serrin, G. Todorova and E. Vitillaro, Existence for a nonlinear wave equation with damping and source terms, Differential Integral Equations 16 (2003), 13-50.

[17] L. Tartar, Existence globale pour un système hyperbolique semi linéaire de la théorie cinétique des gaz, in: Séminaire Goulaouic-Schwartz (1975/1976), Équations aux dérivées partielles et analyse fonctionnelle, exp. no. 1, Centre Math., École Polytech., Palaiseau, 1976, $11 \mathrm{pp}$.

[18] —, Topics in Nonlinear Analysis, Publ. Math. Orsay 7813, Dépt. Math., Univ. de Paris-Sud, Orsay, 1978.

[19] G. Todorova and E. Vitillaro, Blow-up for nonlinear dissipative wave equations in $\mathbb{R}^{n}$, J. Math. Anal. Appl. 303 (2005), 242-257.

Department of Mathematics

University of Nebraska-Lincoln

203 Avery Hall

Lincoln, NE 68588-0130, U.S.A.

E-mail: pradu@math.unl.edu

Received on 14.1.2008;

revised version on 25.3.2008 